PROCEEDINGS OF THE

AMERICAN MATHEMATICAL SOCIETY

Volume 131, Number 10, Pages 3251-3255

S 0002-9939(03)06877-1

Article electronically published on January 8, 2003

\title{
WEAK COMPACTNESS OF CERTAIN SETS OF MEASURES
}

\author{
SURJIT SINGH KHURANA
}

(Communicated by Claudia M. Neuhauser)

\begin{abstract}
For a compact Hausdorff space $X$ and a Montel Hausdorff locally convex space $E$, let $F=(C(X, E), u), u$ being the uniform topology. We determine the necessary and sufficient conditions for an equicontinuous $H \subset F^{\prime}$ to be $\sigma\left(F^{\prime}, F^{\prime \prime}\right)$-compact. Special results are obtained when $X$ is an $F$-space or a $\sigma$-Stonian space.
\end{abstract}

\section{INTRODUCTION AND NOTATIONS}

For locally convex spaces, the notations and results from [14] are used and for general topology we refer to [6]. All vector spaces are taken over $K$, the field of real or complex numbers (we call $K$ the scalar field). For measures and vector measures, we will follow the notations of and results from [2], 7], [8], 9] [13], [16]. For a locally convex space $G, G^{\prime}$ will be its dual. For a completely regular Hausdorff space $Y, \tilde{Y}$ will denote the Stone-Cech compactification of $Y$. N will denote the set of natural numbers. For two vector spaces in duality, $\langle$,$\rangle will denote the bilinear$ form on their product.

In this paper, $X$ is a compact Hausdorff space and $E$ a Montel Hausdorff locally convex space. Recall that a locally convex space is called a Montel space if it is barreled and every bounded set is relatively compact ([10], pp. 369-378). As stated by Köthe ([10], p. 372), some of the most important spaces in analysis are Montel (the space $H(D)$, of analytic functions on the open unit disc, with the topology of uniform convergence on the compact subsets of $D$ is a Fréchet-Montel space ([10], p. 373)). We denote by $C(X, E)$ the space of all $E$-valued continous functions on $X$. If $E=K$, then we denote $C(X, E)$ by $C(X)$. Let $\left\{\|\cdot\|_{p}: p \in P\right\}$ be the collections of all continuous semi-norms on $E$. For a $p \in P, E_{p}$ denotes the normed space arising from $E$ by the semi-norm $\|\cdot\|_{p} . \quad E_{p}^{\prime}$ can be considered a subspace of $E^{\prime}: E_{p}^{\prime}=\left\{f \in E^{\prime}: f\left(p^{-1}[0,1]\right)\right.$ is bounded $\}$. We take the uniform topology $u$ on $C(X, E)$ and denote the locally convex space $(C(X, E), u)$ by $F$. The dual of $F$ is denoted by $M\left(X, E^{\prime}\right)$. If $E$ is a normed space, then with norm topology on $E^{\prime}, M\left(X, E^{\prime}\right)$ consists of regular Borel $E^{\prime}$-valued measures, of finite variations, on $X$. If $E$ is locally convex, then $(C(X, E), u)$ can be considered a subspace of $\prod_{p \in P}\left(C\left(X, E_{p}\right), u\right)$, with induced topology. So for every $\mu \in M\left(X, E^{\prime}\right)$, there

Received by the editors January 2, 2002 and, in revised form, April 28, 2002.

2000 Mathematics Subject Classification. Primary 60B10, 46G10, 46G15, 28C15; Secondary 47B38, 28A51, 54C35.

Key words and phrases. Montel locally convex space, Stonian space, Fréchet space, lifting of measures. 
is a $p \in P$ such that $\mu \in\left(C\left(X, E_{p}\right), u\right)^{\prime}$. Thus every $\mu \in F^{\prime}$ gives a $p \in P$ such that $\mu$ is an $E_{p}^{\prime}$-valued regular Borel measure, of finite variation $|\mu|_{p}$, on $X$ ([8]). If $E=K$, then $M\left(X, E^{\prime}\right)$ is denoted by $M(X)$ and its positive elements are denoted by $M^{+}(X)$. If $H \subset F^{\prime}$ is equicontinuous, then there is a $p \in P$ such that $|\mu|_{p}(X) \leq 1, \forall \mu \in H$, and all $\mu \in H$ are $E_{p}^{\prime}$-valued. We denote by $\gamma$ the canonical mapping $E \rightarrow E_{p}$, by $\mathcal{B}$ all closed, absolutely convex bounded subsets of $E$ (since $E$ is Montel, these subsets are compact), and by $\mathcal{B}_{0}$ the collection $\{\gamma(B): B \in \mathcal{B}\}$; the elements of $\mathcal{B}_{0}$ are absolutely convex and compact in $E_{p}$.

In the celebrated paper of Grothendieck [5], some remarkable characterizations of weakly compact subsets of $M(X)$ are obtained and then are applied to the special case when $X$ is a Stonian space. These results are extended to the case of $C(X, E)$ by many authors (4], [1, [17]). In this paper we give necessary and sufficient conditions for an equicontinuous subset of $F^{\prime}$ to be $\sigma\left(F^{\prime}, F^{\prime \prime}\right)$-compact (i.e. weakly compact in $F_{\beta}^{\prime}$ - note, in the notations of [14, that $F_{\beta}^{\prime}$ is the space $F^{\prime}$ with topology of uniform convergence on the $\sigma\left(F, F^{\prime}\right)$-bounded subsets of $F$ and $\left.\left(F_{\beta}^{\prime}\right)^{\prime}=F^{\prime \prime}\right)$ and then apply the results to the special cases when $X$ is a $\sigma$-Stonian or an $F$-space. Recall that $X$ is Stonian if the closure of every open set is open, is $\sigma$-Stonian if the closure of every open $F_{\sigma}$-set is open, and is $F$-space if disjoint open $F_{\sigma}$-sets have disjoint closures.

We will also need the following lemmas:

Lemma 1. Let $\lambda_{n}: 2^{N} \rightarrow K$ be a sequence of countably additive functions (on all subsets of $N)$. If $\lim \lambda_{n}(A)$ exists for all subsets $A \subset N$, then the convergence is uniform on $2^{N}$.

Proof. This is a particular case of classical Phillips' Lemma ([2], p. 33).

Lemma 2. Let $T$ be a Hausdorff topological space having a $\sigma$-compact dense subset, $C(T)$ all scalar-valued continuous functions on $T$ with the topology of pointwise convergence, and $A \subset C(T)$ such that every sequence in $A$ has a cluster point in $C(T)$. Let $g$ be in the closure of $A$ in $K^{T}$. Then there exists a sequence, in $A$, which converges to $g$ pointwise.

Proof. This is proved in [12].

\section{MAIN RESUlts}

Theorem 3. Let $X$ be a compact set, $E$ a Montel locally convex space and $F=$ $(C(X, E), u)$ (here u denotes the uniform topology). Suppose $H \subset F^{\prime}$ is equicontinuous. Then $H$ is relatively weakly compact in $F_{\beta}^{\prime}$, if and only if, $\forall x \in E$, $\left\{\mu_{x}: \mu \in H\right\}$ is relatively weakly compact in $(M(X),\|\cdot\|)$.

Proof. Suppose $H$ is relatively weakly compact in $F_{\beta}^{\prime}$. Fix an $x \in E$. Since the mapping $\phi:(C(X),\|\|.) \rightarrow(C(X, E), u), f \rightarrow f \otimes x$ is continuous, the adjoint $\phi^{\prime}: F_{\beta}^{\prime} \rightarrow(M(X),\|\|$.$) is continuous and so the result follows.$

Now we come to the converse. Since $H$ is equicontinuous, $H_{0}$, the closed convex hull of $H$ in $F_{\sigma}^{\prime}$, is compact. From this it is easy to verify that $H_{0}$ is complete in $F_{\beta}^{\prime}$. So to prove the weak-compactness of $H$ in $F_{\beta}^{\prime}$, it is enough to prove that every sequence in $H$ has a cluster point ([14], Theorem 11.2). Take a sequence $\left\{\mu_{n}\right\} \subset H$ and let $\bar{H}$ be the closure of $H$ in $F_{\sigma}^{\prime}$. Let $\mu_{0} \in \bar{H}$ be a cluster point $\left\{\mu_{n}\right\}$ in $F_{\sigma}^{\prime}$. There exists a $p \in P$ such that $\left|\mu_{n}\right|_{p}(X) \leq 1,0 \leq n<\infty$. We fix a 
lifting $\rho$ for the finite measure $\lambda=\sum_{n=0}^{\infty} \frac{1}{2^{n+1}}\left|\mu_{n}\right|_{p}$ ([16], [13]). We take the lifting topology $\mathcal{T}_{\rho}$ on $X$ (this topology is denoted by $\mathcal{T}_{1}$ in [13]) and denote the associated completely regular Hausdorff space by $\left(X_{0}, \mathcal{T}_{\rho}\right)$. $\tilde{X}_{0}$ will denote the Stone-Cech compactification of $X_{0}$.

We state some notations and results from [9]:

$M_{0}$ is the linear span of $\mu_{n}, 0 \leq n<\infty$, in $F^{\prime}$.

$V=\left\{x \in E:\|x\|_{p} \leq 1\right\}$.

$L_{1}=\left\{\phi:\left(X_{0}, \mathcal{T}_{\rho}\right) \rightarrow E_{\sigma}^{\prime}, \phi\right.$ continuous and $\left.\phi\left(X_{0}\right) \subset \alpha V^{0}, \alpha>0\right\}$; here $V^{0}$ is the polar of $V$ in the duality $\left\langle E, E^{\prime}\right\rangle$. Since $E$ is Montel, $\phi$ is also continuous in $E_{\beta}^{\prime}$. Also every $\phi \in L_{1}$ is $E_{p}^{\prime}$-valued.

For a $B \in \mathcal{B}, \phi \in L_{1},|\phi|_{B}(t)=\sup \{|\langle x, \phi(t)\rangle|: x \in B\} .|\phi|_{B}$ is continuous on $X_{0}$. Since $\phi$ is $E_{p}^{\prime}$-valued, if we put $B_{0}=\gamma(B)$, then we also have $|\phi|_{B}=$ $\sup \left\{|\langle x, \phi(t)\rangle|: x \in B_{0}\right\}$.

$\{f \in C(X, E): f(X) \subset B\}$, as $B$ varies in $\mathcal{B}$, form a fundamental system of bounded sets in $F$.

For a $\mu \in M_{0}$, there exists a $q \in L_{1}$ such that $\mu=q \lambda$, in the sense that $\mu_{x}=q_{x} \lambda$ (here $q_{x}(t)=\langle x, q(t)\rangle$; also $\sup \{|\mu(f)|: f(X) \subset B\}=\int|q|_{B} d \lambda, \forall B \in \mathcal{B}$ ). Further $|\mu|_{p}(X)=\int|q|_{V} d \lambda$ where $|q|_{V}=\sup \{|x \circ q|: x \in V\}$. From this it follows that $\mu \rightarrow q$ is a linear isomorphism if the topology on $M_{0}$ is the one induced by $F_{\beta}^{\prime}$ and the topology on $L_{1}$ is generated by $\mathcal{B}$ (each $B \in \mathcal{B}$ gives a semi-norm on $\left.L_{1}, q \rightarrow \int|q|_{B} d \lambda\right)$.

$L_{\infty}=\left\{f: X_{0} \rightarrow E_{p}, f\left(X_{0}\right) \subset \gamma(B), B \in \mathcal{B}, f\right.$ continuous with norm topology on $\left.E_{p}\right\}$.

$L_{\infty}=\left(L_{1}, \mathcal{B}\right)^{\prime}$, algebraically.

Now we continue with the proof. Take an $f \in L_{\infty}$. This means there is a $B \in \mathcal{B}$ such that if $B_{0}=\gamma(B)$, then $f\left(X_{0}\right) \subset B_{0}$. By continuity, extend $f$ to $\tilde{f}: \tilde{X}_{0} \rightarrow B_{0}$. Fix $c>0$ and take an $\tilde{f}_{0} \in C\left(\tilde{X}_{0}\right) \otimes E_{p}$ such that $\left\|\tilde{f}-\tilde{f}_{0}\right\|_{p}<c$. Put $\tilde{f}_{0 \mid X_{0}}=f_{0}$. Take a net $\left\{\mu_{\alpha}\right\} \subset\left\{\mu_{n}\right\}$ such that $\mu_{\alpha} \rightarrow \mu_{0}$, in $F_{\sigma}^{\prime}$. Also take a net $\left\{q_{\alpha}\right\} \subset L_{1}$ such that $\mu_{\alpha}=q_{\alpha} \lambda$; further take $q_{0} \in L_{1}$, satisfying $\mu_{0}=q_{0} \lambda$. Since $\int\left|q_{\alpha}\right|_{V} d \lambda \leq 1$ and $\int\left|q_{0}\right|_{V} d \lambda \leq 1$, we have $\int\left|\left\langle f-f_{0}, q_{\alpha}-q_{0}\right\rangle\right| d \lambda \leq c$. Also since $\left\{\left(\mu_{\alpha}\right)_{x}\right\}$ is relatively weakly compact in $(M(X),\|\|$.$) for every x \in E, \int\left\langle f_{0}, q_{\alpha}-q_{0}\right\rangle \mid d \lambda \rightarrow 0$. The result now follows.

Corollary 4. Let $X$ be a compact Hausdorff space, E a Montel-Fréchet locally convex space and $F=(C(X, E), u)$. Suppose $H \subset F^{\prime}$ is bounded in $F_{\sigma}^{\prime}$. Then $H$ is relatively weakly compact, in $F_{\beta}^{\prime}$, if and only if, $\forall x \in E,\left\{\mu_{x}: \mu \in H\right\}$ is relatively weakly compact in $(M(X),\|\|$.$) .$

Proof. In this case $F$ is barreled and so $H$ will be equicontinuous. The result follows from Theorem 3 .

The following theorem is proved in [17]; we give below a different proof.

Theorem 5 ([17, Theorem 3.3, Theorem 3.4]). Let $X$ be a compact Hausdorff space. Assume $X$ is either an $F$-space or a $\sigma$-Stonian space. Suppose $H$ is a compact subset of $(M(X), \sigma(M(X), C(X)))$. The $H$ is weakly compact iff there is a $\lambda \in M^{+}(X)$ such that $\mu<<\lambda$ for every $\mu \in H$.

Proof. Assume that there is a $\lambda \in M^{+}(X)$ such that $\mu<<\lambda$ for every $\mu \in H$. Suppose $H$ is not weakly compact. By ([11], Theorem 2(ii), p. 4846) there is a sequence $\left\{\mu_{n}\right\} \subset H$, a disjoint sequence $\left\{V_{n}\right\}$ of open subsets of $X$, and a $c>0$ 
such that $\left|\mu_{n}\left(V_{n}\right)\right|>c, \forall n$. By regularity, we can assume that each $V_{n}$ is $F_{\sigma}$. Take a sequence $\left\{f_{n}\right\} \subset C(X), 0 \leq f_{n} \leq \chi_{V_{n}}$ such that $\left|\mu_{n}\left(f_{n}\right)\right|>c, \forall n$. Now the $\operatorname{supp}(|\mu|) \subset \operatorname{supp}(\lambda), \forall \mu \in H$. If $X$ is an $F$-space, for notational convenience, we take $X=\operatorname{supp}(\lambda)$ (note that every continuous scalar-valued function on a closed subset of $X$ can be extended to a continuous function on the whole of $X$ ); this means $X$ is Stonian ([15], Theorem 2.2). In both the cases $X$ is $\sigma$-Stonian and so the real-valued functions in $C(X)$ are an order $\sigma$-complete vector lattice. For any $M \subset N$, let

$$
f_{\mid M}=\sup _{i n C(X)}\left\{\sum_{i \in M, i \leq n} f_{i}: n \in N\right\}
$$

(for $M=\emptyset, f_{\mid M}=0$ ). It is easy to verify that if $M_{1} \cap M_{2}=\emptyset$, then $f_{\mid M_{1}} \wedge f_{\mid M_{2}}=0$. Define $\lambda_{0}: 2^{N} \rightarrow R^{+}, \lambda_{0}(M)=\lambda\left(f_{\mid M}\right)$. $\lambda_{0}$ is finitely additive and exhaustive ([3] $)$ and $\lambda_{0}(\{n\})=\lambda\left(f_{n}\right), \forall n$. By ([3], Proposition 1, p. 728), there is an infinite subset of $N$, which again we denote by $N$, such that $\lambda_{0}$ is countably additive on $2^{N}$. Bringing this information on $\lambda$, we get $\lambda\left(f_{\mid M}-\sum_{i \in M} f_{i}\right)=0, \forall M \subset N$, and so for every $\mu \in H,|\mu|\left(f_{\mid M}-\sum_{i \in M} f_{i}\right)=0, \forall M \subset N$. Define $\nu_{n}: 2^{N} \rightarrow$ $K, \nu_{n}(M)=\mu_{n}\left(\sum_{i \in M} f_{i}\right)=\mu\left(f_{\mid M}\right)$. These measures are countably additive. Let $Y=\left\{f_{\mid M}: M \subset N\right\}$ be given the topology induced by $K^{H}$ with product topology. This means the countable set $\left\{f_{\mid M}: M\right.$ a finite subset of $\left.N\right\}$ is dense in $Y$. Applying Lemma 2 to $H \subset C(Y)$ (note that $H$ is playing the role of $A$ in Lemma 2), we get a subsequence of $\left\{\mu_{n}\right\}$, which again we denote by $\left\{\mu_{n}\right\}$, and a $\mu_{0} \in H$ such that $\mu_{n} \rightarrow \mu_{0}$, pointwise on $Y$. This implies that $\lim \nu_{n}(M)$ exists for any $M \subset N$. By Lemma $1, \mu_{n}\left(f_{n}\right) \rightarrow 0$ which is a contradiction.

The converse is trivial.

Theorem 6. Let $X$ be a compact Hausdorff space. Assume that $X$ is also either an F-space or a $\sigma$-Stonian space. Let $E$ be a Montel locally convex space and $F=(C(X, E), u)$ (here $u$ denotes the uniform topology). Suppose $H \subset F^{\prime}$ is equicontinuous and $F_{\sigma}^{\prime}$-closed. Then $H$ is relatively weakly compact in $F_{\beta}^{\prime}$, if and only if, for every $x \in E$, there exists a $\lambda_{x} \in M^{+}(X)$ such that $\mu_{x}<<\lambda_{x}$, for every $\mu \in H$.

Proof. Suppose the condition of the theorem is satisfied. Fix an $x \in E$. By Theorem 3 , we have only to prove that $\left\{\mu_{x}: \mu \in H\right\}$ is relatively weakly compact in $M(X)$. The mapping $F_{\sigma}^{\prime} \rightarrow(M(X), \sigma(M(X), C(X))),\left(\mu \rightarrow \mu_{x}\right)$ is continuous and so $\left\{\mu_{x}: \mu \in H\right\}$ is $\sigma(M(X), C(X))$-compact. The result follows from Theorem 5 .

We are very grateful to the referee for some very useful suggestions which have improved the paper.

\section{REFERENCES}

[1] Brooks, J. K., Lewis, P. W., Linear operators and vector measures, Trans. Amer. Math. Soc. 192(1974), 139-162. MR 49:3585

[2] Diestel, J., Uhl, J. J., Vector Measures, Amer. Math. Soc. Surveys, Vol. 15, Amer. Math. Soc., 1977. MR 56:12216

[3] Drewnowski, L., Equivalence of Brooks-Jewett, Vitali-Hahn-Saks and Nikodym Theorems, Bull. Acad. Polon. Sci. Ser. Math. Astronom. Phys. 20(1972), 725-731. MR 47:431

[4] Freniche, F. J., Grothendieck locally convex spaces of continuous vector-valued functions, Pac. J. Math. 120(1985), 345-355. MR 87a:46059

[5] Grothendieck, A., Sur les applicationes lineares faiblement compactes d'espace du type C(K), Can. J. Math. 5(1953), 129-173. MR 15:438b 
[6] Kelley, J. L., General Topology, Van Nostrand, Princeton, N. J., 1955. MR 16:1136c

[7] Khurana, S. S., Topologies on spaces of vector-valued continuous functions, Trans. Amer. Math. Soc. 241(1978), 195-211. MR 81f:46053

[8] Khurana, S. S., Topologies on spaces of vector-valued continuous functions II, Math. Ann. 234(1978), 159-166. MR 82f:46042

[9] Khurana, S. S., Grothendieck Spaces, II, J. Math. Anal. Appls. 159(1991), 202-207. MR 92i: 46042

[10] Köthe, G., Topological vector spaces I, Springer-Verlag, New York, 1969. MR 40:1750

[11] Panchapagesan, T. V., Baire and $\sigma$-Borel characterizations of weakly compact sets in $M(T)$, Trans. Amer. Math. Soc. 350(1998), 4839-4847. MR 99h:28008

[12] Pryce, J. D., A device of R. J. Whitley applied to pointwise compactness in spaces of continuous functions, Proc. Lon. Math. Soc. 23(1971), 532-546. MR 45:5729

[13] Rao, M. M., Measure Theory and integration, John Wiley \& Sons, New York, 1987. MR 89k:28001

[14] Schaefer, H. H., Topological vector spaces, Springer-Verlag, 1970. MR 49:7722

[15] Seever, G. L., Measures on F-spaces, Trans. Amer. Math. Soc. 133(1968), 267-280. MR 37:1976

[16] Ionescu Tulcea, A., Ionescu Tulcea, C., Topics in the theory of liftings, Springer-Verlag, New York, 1969. MR 43:2185

[17] Zhang, Xiao-Dong, On weak compactness in spaces of measures, J. Funct. Anal. 143(1997), 1-9. MR 98d:46030

Department of Mathematics, University of Iowa, Iowa City, Iowa 52242

E-mail address: skhurana@blue.weeg.uiowa.edu 